\title{
Converting Human Thoughts into Text through Mind Gate and Mind Computer Interface Devices
}

\author{
Mahesh Nelapati ${ }^{1}$, Naresh Madavarapu ${ }^{2}$ \\ Assistant Professor, IT Department, Gurunanak Institute of Technology, Hyderabad, Telangana, India ${ }^{1,2}$
}

\begin{abstract}
The Brain Gate System is set up on Cyber energy organize innovation to detect, exchange, look at and put on the language of neurons. The standard of activity behind the Brain Gate System is that with unblemished cerebrum work, mind signals are created despite the fact that they are not sent to the arms, hands and legs. The signs are deciphered and converted into cursor developments, offering the client another Brain Gate pathway to control a PC with thought, similarly as people who can move their hands utilize a mouse. The 'Mind Gate' contains minor spikes that will reach out down around one millimetre into the cerebrum subsequent to being embedded underneath the skull, checking the action from a little gathering of neurons. It will presently be workable for a patient with spinal rope damage to make cerebrum flags that pass on the aim of moving the deadened appendages, as signs to an embedded sensor, which is then yield as electronic driving forces. These driving forces help the client to work mechanical gadgets with the assistance of a PC cursor. Matthew Nagle, a 25-year-old Massachusetts individual with unadorned spinal string harm, has been incapacitated starting from the neck since 2001[1].After partaking in a clinical preliminary of this framework, he has opened email, exchanged TV channels, turned on lights [2]. He even moved an automated hand from his wheelchair. This character the first occasion when that neural development signals have been recorded and decoded in a human with spinal rope damage. The framework is additionally the first to allow a human to direct his close-by circumstance utilizing his psyche.
\end{abstract}

Keywords: Neurons, Brain Gate, cerebrum flags, Neural Development Signals

\section{INTRODUCTION}

Mind Gate framework was built up by means of the bio-tech organization Cyber energy in 2003 in Combination with the bureau of Neuroscience Brown University [3].

The gadget was intended to enable the individuals who to have lost control of their appendages or other body work. The PC chip which is fixed into the mind, screens cerebrum activity in the patient and adjust the intension of the client into PC hands. As of now the chip utilized 100 hair-slim terminals that hear neurons terminating in explicit territory of the mind. For e.g.: the part that control the arm development the activity is deciphered into variedly Charged flags and are then set and deciphered utilizing a program in this way influencing the arm. As indicated by the Cyber energy site, two patients have been embedded with the Brain Gate. In November 2011, specialists from the Stanford University Neural Prosthetics Translational Laboratory joined the preliminary as a second site. [4][5].

The Brain Gate System depends On "Digital energy" stage advances to detect, transmit, dissect and apply the language of neurons. The System includes a sensor that is fixed on the engine cortex of the cerebrum and a gadget that looks at mind signals. The guideline of activity behind the Brain Gate System is that with flawless cerebrum work, mind signals are produced despite the fact that they are not sent to the arms, hands and legs. The signs are comprehended and converted into cursor developments, offering the client an improved "Mind Gate pathway." To control a PC with thought, similarly as people who can move their hands utilize a mouse.

\section{NEUROMOTOR PROSTHETIC DEVICE}

A neuro prosthetic gadget known as Brain door changes mind activity into PC rules. A sensor is secure on the mind, and cathodes are snared to wires that helpful to a platform on the scalp. From that point, a fiber optic link exchanges the cerebrum development information to a neighbouring computer. 


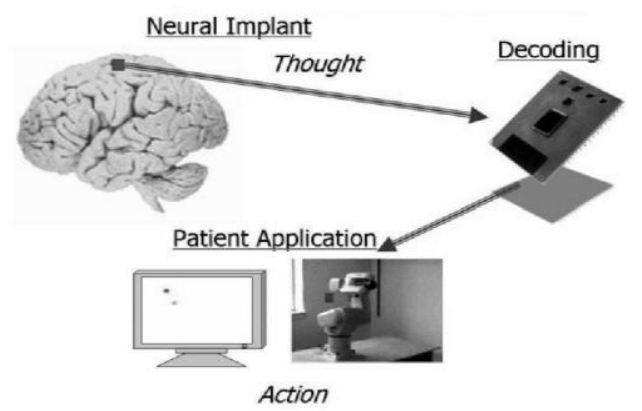

Fig 1: Neuro motor prosthetic device

\section{PRINCIPLE}

The rule of method of the Brain Gate Neural Interface System is that with entire mind work, neural signs are delivered despite the fact that they are not sent to the arms, hands and legs. These signs are comprehended by the System and a cursor is appeared to the client on a PC screen that offers a substitute "Cerebrum Gate pathway". The administrator can utilize that cursor to direct the PC, similarly as a mouse is utilized [6].

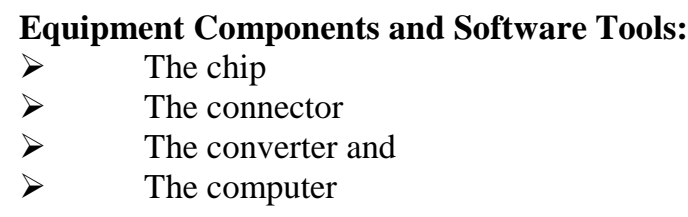

$>\quad$ The Chip:

A 4-millimeter square silicon chip specked with hundred hair-meagre, microelectrodes is installed in cerebrum essential engine cortex.

The chip, around the extent of an infant ibuprofen, holds 100 anode sensors, every more slender than a human hair. The sensors sense minute electrical signs delivered when a client imagines, for instance, that he's influencing the bolt, its producer talks.

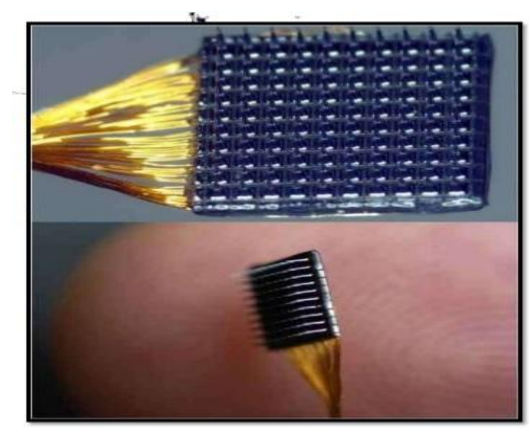

Fig 2: Brain Gate Chip

In spite of the fact that incapacitated, a quadriplegic calm has the ability to create such flags - they simply don't gain past the harmed bit of the spinal line. With Brain Gate, the signs in its place portable through a wire that leaves the skull and connections to a PC, Cybernetics says. Cerebrum Gate utilizes innovation like cochlear inserts that assistance hard of hearing individuals hear and profound mind test systems that treat Parkinson's malady. Those gadgets cost $\$ 15,000$ to $\$ 25,000$. Mind Gate will be "in any event that costly, and maybe more", Surgeon said.

\section{$>\quad$ The Connector:}

It is dedicated undauntedly to the skull of the patient and it allows the signs gotten by the chip to the converter. Most debilitation individuals are fulfilled. In the event that they can get a fundamental association with the protected world. Mind Gate empowers them to accomplish unmistakably more than that. Through checking the PC cursor, patients can get to web measurements, TV excitement, and direct lights and uses — with simply their considerations [7] [8].

What's more, as this stunning innovation advance\% analysts trust it could empower cerebrum signs to sidestep harmed nerve tissues and re-establish portability to incapacitate appendages. "The objective of Brain Gate is to build up a quick, solid, and unpretentious association between the mind of a seriously cripple individual and a PC" said Cyber energy Leader group Surgeon. 


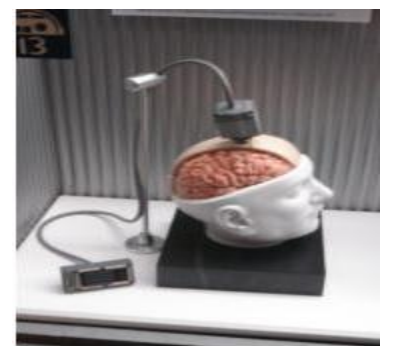

Fig 3: Brain Gate Connector

Mind Gate may seem like sci-fi, however it's definitely not. The gadget is irrelevant than a dime and includes 100 wires littler than human hairs which associate with the bit of the cerebrum that controls engine movement. The wires sense when neurons are energized and coordinates those signs during a time connector mounted masterminded the skull to a PC.

The converter:

The flag goes through a shoebox-sized intensifier where it's changed to Digital information and pulled back by fiberoptic link to a PC.

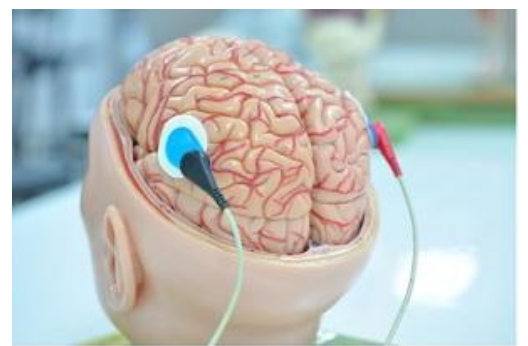

Fig 4: Brain Gate Converter

The Computer:

Mind Gate concentrates to help aim of cerebrum development with specific envisioned developments up, down, left, right and to associate those developments to a cursor [9].

A mind PC interface uses electro physiological signs to direct detached gadgets [10]. Most present BCIs are not obtrusive. They comprise of terminals connected to the scalp of an individual or harmed in a cathode top, for example, the one appeared in 1-1 (Left). Every one of these conduits get the braincases electrical movement (at the microvolt level) and convey it into intensifiers, for example, the ones appeared in 1-1 (Right). These enhancers intensify the flag around ten thousand times and after that license the flag through a simple to advanced converter to a PC for preparing. The PC Processes the EEG flag and uses it in direction to achieve undertakings, for example, correspondence and ecological control. Bps is moderate in examination with ordinary human activities, on account of the intricacy andtumult [11].

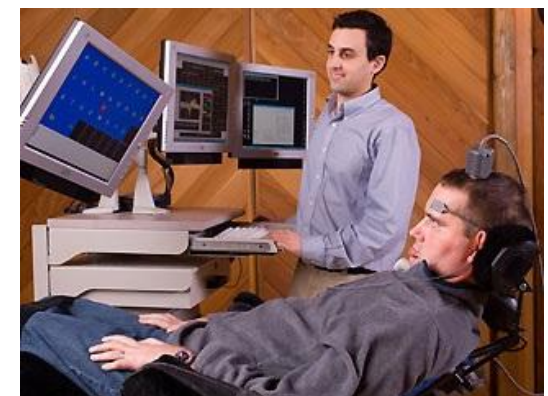

Fig 5: Brain Gate Computer

\section{SOFTWARE BEHIND BRAINGATE}

Programming on which BRAINGATE System works utilizes calculations and example coordinating techniques to encourage correspondence. The calculations are written in C, JAVA and MATLAB. The flag preparing programming calculations look at the electrical development of neurons and translate it into control signals implied for use in different PC based applications. One member, cathy Hutchinson, had the capacity to utilize the arm to drink espresso from jug [12]. 


\section{WORKING}

The basic structure of a Brain-Computer Interface is the accompanying:

$>\quad$ Signal Gaining: The EEG signals are acquired from the cerebrum through obtrusive or non-intrusive strategies [13] (for instance, anodes).

$>\quad$ Signal Pre-Processing: When the signs are accomplished, it is necessary to clean them.

$>\quad$ Signal Grouping: when the signs are cleaned, they will be handled and characterized to discover which sort of mental undertaking the subject is practiced.

$>\quad$ Computer Communication: when the signs are characterized, they will be utilized by an appropriate calculation for some application [16].

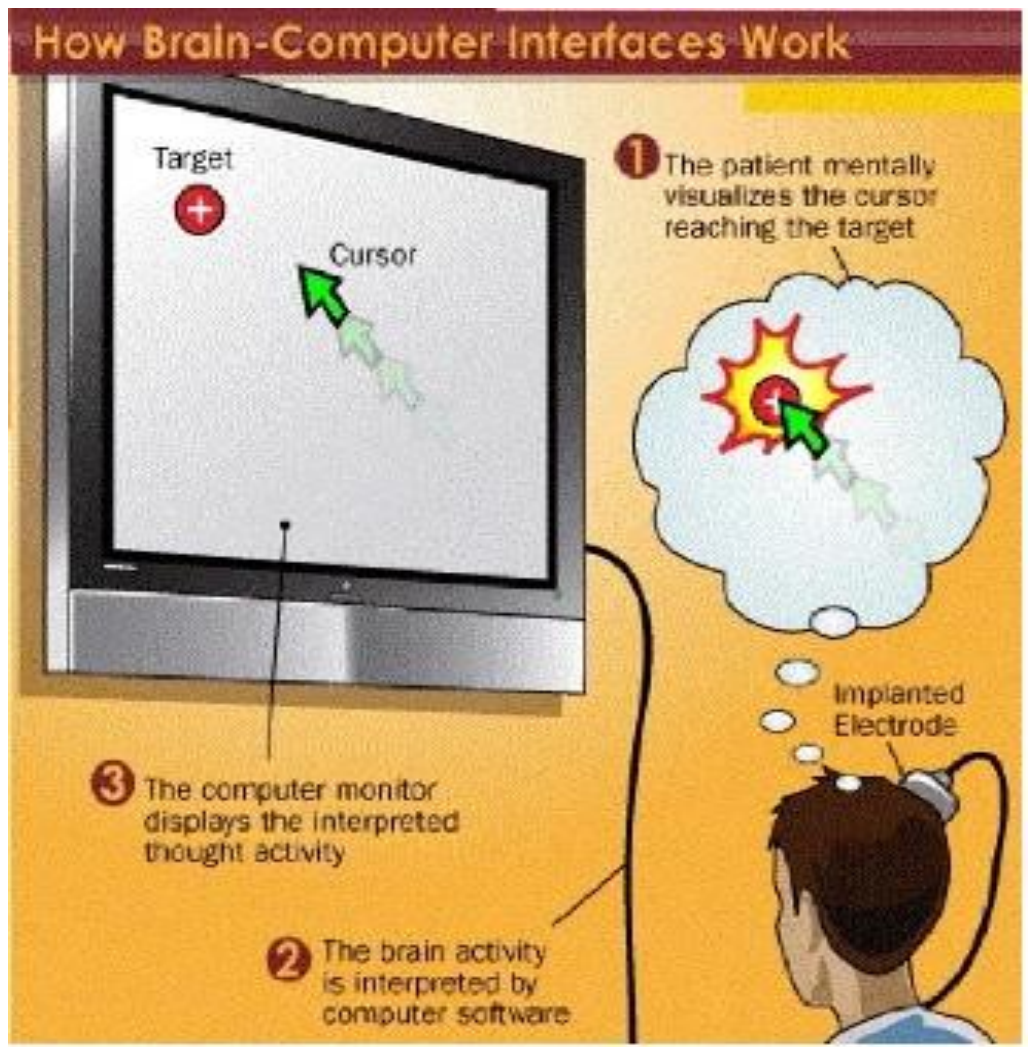

Fig 6: Brain Gate Working process

\section{APPLICATIONS}

Navigate Internet

Play Computer Games

Turn Lights On and Off

Control Television

Control Robotic Arm

\section{ADVANTAGES}

Brain Gate can remain securely embedded in the mind for something like two years.

$>$ Later it can securely be evacuated too.

$>$ Spiking from numerous neurons the language of the cerebrum can be recorded, steered outside the human mind and decoded into order signals.

$>$ Paralyzed people can straightforwardly and effectively control outer gadgets, for example, a PC cursor utilizing these neural direction signals.

$>$ The speed, exactness, and accuracy are practically identical to non-handicapped individual there is no preparation vital (simply the capacity to think about an activity). 


\section{DRAWBACKS}

One detriment is that the brain gate chip requires to be embedded inside the patient's cerebrum. This is a noteworthy drawback in view of if the strategy does not go as plan or in the event that the specialist goofs on the grounds that no human is immaculate, at that point an individual could bite the dust or get cerebrum harm. An option could be utilizing an electromagnetic top for the individual to wear despite the fact that it leaves something to be desired as the chip. The advancement could have been additionally inquired about. Another weakness of this innovation would be on the grounds that it if not remote good yet, every individual that has this brain gate chip embedded into the cerebrum should be associated with a seat by means of a link that has the PC incorporated with it. So brain gate isn't yet free development nor is it substantial developments yet. An individual uses an automated arm to do the majority of their developments at present. Specialists are facilitating the arm to deal with progressively convoluted developments, for example, brushing teeth.

Where there were victors of this innovation being created and promoted there are washouts also. The failures of this innovation could be the all inclusive community; this is on the grounds that not every person is rich and can bear the cost of the expenses. By and large when another innovation is discharged, the value purpose of that innovation is quite steep for anybody however the rich to manage. Another conceivable weakness is conceivable reactions of the chip being in the mind. What occurs if the chip over warms or yields/inputs a lot of an electrical flow from the chip. It is obvious from the above sections that there are points of interest to the brain gate innovation and yet there are detriments as you have perused. This is a cycle that will keep on going on as long as new innovation is designed.

\section{CONCLUSION}

The knowledge of moving robots or prosthetic devices not by physical control, but by meagre "thinking" (i.e., the brain activity of human subjects) has been a captivated method. Therapeutic treatments are inaccessible for numerous resources of neural and muscular paralysis [15]. The fear of the arrears caused by paralysis is a robust motivation to follow BMI solutions. So this indication supports numerous patients to regulate the prosthetic devices of their separate by simply thinking about the task.

This technology is well supported by the latest fields of Biomedical Instrumentation, Microelectronics signalprocessing, Artificial Neural Networks and Robotics which has overwhelming developments [16]. Expected these systems will be effectively applied for many biomedical applications.

\section{REFERENCES}

[1]. "Mind Control". Wired. March 2005.

[2]. Hochberg LR, Serruya MD, Friehs GM, Mukand JA, Saleh M, Caplan AH, Branner A, Chen D, Penn RD, Donoghue JP (July 2006). "Neuronal ensemble control of prosthetic devices by a human with tetraplegia". Nature. 442 (7099): 164-171. doi:10.1038/nature04970. PMID 16838014.

[3]. Brain Gate gets a new lease on life, The Boston Globe, August, 2009

[4]. "Stanford joins Brain Gate team developing brain-computer interface to aid people with paralysis". Stanford. November 11, 2011.

[5]. TANYA LEWIS. "Stanford joins Brain Gate team developing brain-computer interface to aid people with paralysis". Stanford School of Medicine. Archived from the original on December 9, 2011.

[6]. Hochberg LR, Bacher D, Jarosiewicz B, Masse NY, Simeral JD, Vogel J, Haddadin S, Liu J, Cash SS, van der Smagt P, Donoghue JP (May 2012). "Reach and grasp by people with tetraplegia using a neurally controlled robotic arm". Nature. 485 (7398): $372-375$. doi:10.1038/nature11076. PMC 3640850Freely accessible. PMID 22596161.

[7]. "People with paralysis control robotic arms using brain-computer interface". Brown University. May 2012.

[8]. "Mind-controlled robot arms show promise". Nature. May 16, 2012.

[9]. "The Annual BCI Research Award 2014 - The Winners". Biosignal.at. 15 June 2011. Retrieved 19 December 2016.

[10]. Do, an H; Wang, Po T; King, Christine E; Chun, Sophia N; Nenadic, Zoran (2013). "Brain-computer interface controlled robotic gait orthosis". Journal of NeuroEngineering and Rehabilitation

[11]. Höhne, J; Holz, E; Staiger-Sälzer, P; Müller, KR; Kübler, A; Tangermann, M (2014). "Motor imagery for severely motor- impaired patients: evidence for brain-computer interfacing as superior control solution.

[12]. "People with paralysis control robotic arms using brain-computer interface". Stanford University. May 2012.

[13]. Gallegos-Ayala, G; Furdea, A; Takano, K; Ruf, CA; Flor, H; Birbaumer, N (27 May 2014). "Brain communication in a completely locked-in patient using bedside near-infrared spectroscopy".

[14]. "The boston home connection to a technology breakthrough". May 16, 2012.1

[15]. "Direct brain-to-brain communication demonstrated in human subjects". Science Daily. 3 September 2014. Retrieved 9 September 2014

[16]. "A Study on Brain Gate Technology for Bio Medical Applications",02,Dec,2017. 